\title{
Cardinal Coordinate Independence for Expected Utility
}

\author{
Peter Wakker
}

University Nijmegen

\begin{abstract}
A representation theorem for binary relations on $\mathbb{R}^{n}$ is derived. It is interpreted in the context of decision making under uncertainty. There we consider the existence of a subjective expected utility model to represent a preference relation of a person on the set of bets for money on a finite state space. The theorem shows that, for this model to exist, it is not only necessary (as has often been observed), but it also is sufficient, that the appreciation for money of the person has a cardinal character, independent of the state of nature. This condition of cardinal appreciation is simple and thus easily testable in experiments. Also it may be of help in relating the neo-classical economic interpretation of cardinal utility to the von Neumann-Morgenstern interpretation.
\end{abstract}

\section{INTRODUCTION}

In this paper a characterization of the subjective expected utility (SEU) model is given. To this end in Section 2 a representation theorem for binary relations on $\mathbb{R}^{n}$ is given. It is based mainly on Debreu (1960), or Chapter 6 of Krantz, Luce, Suppes, and Tversky (1971). The new characterizing property, denoted by CCI, is simple, and yields a handy, empirically testable, criterion. The question what such a property is, has more or less been posed (in the footnote on p. 19 of Marschak and Radner (1972), Section 7.2 of Fishburn (1970), and as Formula 2.7 of Drèze (1982). It leads to a cardinal utility function in the von Neumann-Morgenstern sense; i.e., its stochastic expected value is used to value bets. It may also appeal to the neo-classical economic interpretation, where cardinal utility is a psychological primitive, indicating strength of preference.

In Sarin (1982) and Fishburn (1970, Theorem 7.4) a quaternary relation $\succcurlyeq *$, comparing preference differences, is introduced as a primitive. Then, in terms of $\geqslant *$, conditions are given under which a SEU model exists, such that it agrees with $\geqslant *$. We take as the point of departure that only the preference relation $\geqslant$ on the set of acts is observable. (In Section 3 we use the term "bets" instead of "acts.") A strength of preference relation $\geqslant *$ may exist, but is not considered directly observable. Therefore our characterization is directly in terms of $\geqslant$. With the notion of strength of preference in mind, one will not be surprised by the form of our new characterizing

The presentation of the paper improved by helpful suggestions of F. A. J. Birrer and the referees. Formalizing strength of preference by means of a quaternary relation, as Sarin (1982) did, was suggested by a referee. Requests for reprints should be sent to Dr. Peter Wakker, Department of Mathematics, University Nijmegen, Toernooiveld, 6525 ED Nijmegen, The Netherlands. 
property. It guarantees the possibility of deriving from $\geqslant a$ strength of preference relation for money, independent of the state of nature. Thus the condition is not only necessary for the existence of a SEU model (as has often been observed), but it also is sufficient, in the present context.

Most common, in characterizations of SEU, is the approach, originating from von Neumann and Morgenstern (1944, 1947, 1953, Section I.3 and appendix), where lotteries on the set of acts are introduced. See, for instance, Fishburn (1982). If act $x$ is preferred to a lottery that with probability $1 / 2$ yields act $y$, and with probability $1 / 2$ act $z$, then the strength of preference between $x$ and $y$ should exceed that between $z$ and $x$. In Segal (1982) the case is considered where there is an upper bound for the number of prizes of lotteries.

In Camacho (1980) a preference relation on sequences of acts (maintaining terminology) is considered. If twice act $x$ is preferred to once act $y$ and once act $z$, then the strength of preference between $x$ and $y$ should exceed that between $z$ and $x$. Thus in both mentioned approaches strength of preference directly derives from an extra notion.

In de Finetti (1937) the SEU model is derived, with the (implicit) assumption that the bets, considered there, can be summed. There, if bet $x$ is preferred to $y$, then $x+v$ should be preferred to $y+v$ for all $v$. Then again, if $x+y$ is preferred to $z+w$, then the strength of preference between $x$ and $z$ exceeds that between $w$ and $y$. (This will come down to the special case of our Theorem 3.1 where $U$ is identity.)

The best known derivation of the SEU model is in the first five chapters of Savage (1954). Here only the preference relation $\geqslant$ on the set of acts is taken as primitive, and no strength of preference relation is brought in "from outside" by some extra notion. This has also been our aim. (Contrary to what is often thought, in Savage's model the state space can be denumerable. For other misunderstandings w.r.t. Savage (1954), see Wakker (1981).)

For surveys on SEU characterizations see Fishburn (1981) or Schoemaker (1982).

\section{A Representation Theorem}

This section will be technical. First some notations and definitions are introduced. Elements of $\mathbb{R}^{n}$ are denoted by $a, v, x$, etc., with coordinates $a_{1}, x_{2}$, etc. Elements of $\mathbb{R}$ are denoted by Greek characters, $\alpha, \beta$, etc. ( $\phi$ excepted). A notation that is less standard is the following: $\left(x_{-i} \alpha\right)$ is the element of $\mathbb{R}^{n}$ with $i$ th coordinate $\alpha$, other coordinates equal to those of $x ;\left(x_{-i, j} \alpha, \beta\right)$ is the element of $\mathbb{R}^{n}$ with ith coordinate $\alpha$, $j$ th coordinate $\beta$, other coordinates equal to those of $x$. For a binary relation $\geqslant$ we write $x \geqslant y$ instead of $(x, y) \in \geqslant$. For the usual properties of binary relations the reader is referred to the references. Some more notation, $x \leqslant y$ if $y \geqslant x, x<y$ if not $x \geqslant y, x>y$ if not $x \leqslant y, x \approx y$ if $x \geqslant y$ and $y \geqslant x$. A weak order $\geqslant$ is total $(x \geqslant y$ or $y \geqslant x$ for all $x, y$ ) and transitive, inducing an equivalence relation $\approx$. Coordinate $i$ is essential (w.r.t. $\geqslant)$ if $\left(x_{-i} \alpha\right)>\left(x_{-i} \beta\right)$ for some $x, \alpha, \beta$. We say $\geqslant$ is monotone if $x \geqslant y$ whenever $x_{j} \geqslant y_{j}$ for all $j$, and $\geqslant$ is continuous if $\{x \mid x \geqslant y\}$ and $\{x \mid x \leqslant y\}$ are closed for all $y$. 
DEFINITION. We say $\succcurlyeq$ is coordinate independent $(C I)$ if

$$
\left(x_{-i} \alpha\right) \geqslant\left(y_{-i} \alpha\right) \Rightarrow\left(x_{-i} \beta\right) \geqslant\left(y_{-i} \beta\right) \text { for all } x, y, i, \alpha, \beta \text {. }
$$

DEFINITION. We say $\geqslant$ is cardinally coordinate independent $(C C I)$ if for all $x, y$, $v, w, \alpha, \beta, \gamma, \delta, j$, and essential $i$, from $\left(x_{-i} \alpha\right) \leqslant\left(y_{-i} \beta\right) \&\left(x_{-i} \gamma\right) \geqslant\left(y_{-i} \delta\right) \&$ $\left(v_{-j} \alpha\right) \geqslant\left(w_{-j} \beta\right)$ follows $\left(v_{-j} \gamma\right) \geqslant\left(w_{-j} \delta\right)$.

Elucidation. Replacement of $(\alpha, \beta)$ by $(\gamma, \delta)$ changes $\leqslant$ into $\geqslant$, from the first preference to the second. We imagine this replacement should thus kind of "reinforce" $\geqslant$ from the third to the fourth preference. So certainly not should it change $\geqslant$ into $<$, from the third to the fourth preference.

Definition. We say $\geqslant$ is standard sequence invariant if for all $x, y, \mu, v, \sigma, \tau$, $i \neq k, j \neq l$, with $i$ essential, the relation $\left(y_{-j, l} \beta, \sigma\right) \approx\left(y_{-j, l} \gamma, \tau\right)$ is implied by the relations $\left(x_{-i, k} \alpha, \mu\right) \approx\left(x_{-i, k} \beta, v\right), \quad\left(x_{-i, k} \beta, \mu\right) \approx\left(x_{-i, k} \gamma, v\right), \quad$ and $\quad\left(y_{-j, l} \alpha, \sigma\right) \approx$ $\left(y_{-j, l} \beta, \tau\right)$.

This property is (a minor variation on) the condition in Theorem 15(i) of Section 6.11.2 of Krantz et al. (1971). Their Axiom 5 in Section 8.2.6, formulated in a complex structure, comes closest to our CCI.

For the case $n=2$ we shall need one more property.

Definition. If $n=2$, then $\geqslant$ satisfies the Thomsen-Blaschke $(T B)$ condition if the relation $(\gamma, \mu) \approx(\alpha, v)$ follows from $(\alpha, \mu) \approx(\beta, v), \quad(\alpha, v) \approx(\beta, \sigma), \quad$ and $(\gamma, v) \approx(\alpha, \sigma)$, for all $\alpha, \ldots, \sigma$.

Lемма 2.1. If $\approx$ is an equivalence relation, then $x \approx y$ whenever $x_{j}=y_{j}$ for all essential $j$.

Proof. As an example, let $1,2,3$ be not essential, $x_{j}=y_{j}$ for all $j \geqslant 4$. Then $x \approx\left(x_{-1} y_{1}\right) \approx\left(x_{-1,2} y_{1}, y_{2}\right) \approx\left(\left(x_{-1,2} y_{1}, y_{2}\right)_{-3} y_{3}\right)=y$.

LEMMA 2.2. If $\approx$ is an equivalence relation, then $C C I$ implies $C I$.

Proof. If no coordinate is essential this follows from Lemma 2.1. Otherwise reflexivity of $\geqslant$ is sufficient. To see this, let $\beta \in \mathbb{R},\left(x-{ }_{j} \alpha\right) \geqslant\left(y_{-j} \alpha\right)$. Let $i$ be essential. Then apply CCI with $\left(v_{-i} \alpha\right) \leqslant\left(v_{-i} \alpha\right),\left(v_{-i} \beta\right) \geqslant\left(v_{-i} \beta\right),\left(x_{-j} \alpha\right) \geqslant\left(y_{-j} \alpha\right)$, yielding $\left(x_{-j} \beta\right) \geqslant\left(y_{-j} \beta\right)$. Here $v$ is arbitrary.

LEMMA 2.3. CCI implies standard sequence invariance.

Proof. It is logically stronger.

LEMMA 2.4. If $\approx$ is an equivalence relation, or if at least one coordinate is essential, then CCI implies TB. (For $n=2$.) 
Proof. Let $n=2$. If no coordinate is essential Lemma 2.1 applies. Next let coordinate 1 be essential. Let $i=j=1,(\alpha, v) \leqslant(\beta, \sigma),(\gamma, v) \geqslant(\alpha, \sigma),(\alpha, \mu) \geqslant(\beta, v)$, then $\mathrm{CCI}$ yields $(\gamma, \mu) \geqslant(\alpha, v)$. Exchanging in these preferences first and second bet gives $(\beta, \sigma) \leqslant(\alpha, v),(\alpha, \sigma) \geqslant(\gamma, v),(\beta, v) \geqslant(\alpha, \mu)$, so $(\alpha, v) \geqslant(\gamma, \mu)$ by CCI. Thus CCI implies TB. If coordinate 2 is essential a symmetric argument applies.

We are now ready for our main theorem.

THEOREM 2.1. Let $\geqslant$ be a binary relation on $\mathbb{R}^{n}$. Then the following (i) and (ii) are equivalent:

(i) There exist nonnegative $\left(\lambda_{j}\right)_{j=1}^{n}$ and a continuous function $U: \mathbb{R} \rightarrow \mathbb{R}$, such that $x \geqslant y \Leftrightarrow \sum_{j=1}^{n} \lambda_{j} U\left(x_{j}\right) \geqslant \sum_{j=1}^{n} \lambda_{j} U\left(y_{j}\right)$ for all $x, y$.

(ii) $\succcurlyeq$ is a continuous weak order that satisfies CCI.

Furthermore, if (i) applies, and two or more coordinates are essential, then $U$ is an interval scale (i.e., can be replaced by $U^{\prime}$ if and only if $\beta$ and positive $\alpha$ exist such that $\left.U^{\prime}=\alpha U+\beta\right)$.

Proof. It is straightforward that (i) implies (ii). Next we assume (ii), and derive (i). If no coordinate is essential, then by Lemma 2.1 we see that $x \approx y$ for all $x, y$; and everything follows. If one coordinate, say $i$, is essential, then we apply Section 4.6 of Debreu (1959). This guarantees existence of a continuous function $\phi: \mathbb{R}^{n} \rightarrow \mathbb{R}$, such that for all $x, y$ we have $x \geqslant y \Leftrightarrow \phi(x) \geqslant \phi(y)$. Then let $\phi(x)=U\left(x_{i}\right), \lambda_{i}=1, \lambda_{j}=0$ for all $j \neq i$.

If two or more coordinates are essential, then first we guarantee existence of continuous functions $\left(V_{j}\right)_{j=1}^{n}$, such that $\left[x \geqslant y \Leftrightarrow \sum_{j=1}^{n} V_{j}\left(x_{j}\right) \geqslant \sum_{j=1}^{n} V_{j}\left(y_{j}\right)\right]$ for all $x, y \in \mathbb{R}^{n}$. If exactly two coordinates are essential, then we leave out the other coordinates. This may be done by Lemma 2.1 ; the $V_{j}$ 's associated with the removed coordinates $j$ are taken constant. As indicated in Debreu (1960), the "Hauptsatz über Sechseckgewebe" of Section 1.2 of Blaschke and Bol (1938) guarantees the existence of $\left(V_{j}\right)_{j=1}^{n}$ as above, for any continuous weak order on $\mathbb{R}^{2}$ that is $\mathrm{CI}$ and $\mathrm{TB}$, and has both coordinates essential. CI and TB of $\geqslant$ have been guaranteed by our Lemmas 2.2 and 2.4 .

If three or more coordinates are essential, then we apply Theorem 3 of Debreu (1960). This guarantees existence of $\left(V_{j}\right)_{j=1}^{n}$ as above, for any continuous weak order on $\mathbb{R}^{n}$ that is $\mathrm{CI}$, and has at least three coordinates essential. $\mathrm{CI}$ of $\geqslant$ has been guaranteed by our Lemma 2.2 .

So, if two or more coordinates are essential, then $\left(V_{j}\right)_{j=1}^{n}$ as above exist. By Lemma 2.3 we see that $\geqslant$ is standard sequence invariant. By Theorem 15 of Section 6.11.2 of Krantz et al. (1971) this is (necessary and) sufficient for the existence of real numbers $\left(\lambda_{j}\right)_{j=1}^{n}$, and a continuous $U$, such that $V_{j}=\lambda_{j} U$ for all $j$. Since CCI excludes $\left(x_{-i} \alpha\right)>\left(x_{-i} \beta\right) \&\left(y_{-j} \beta\right)>\left(y_{-j} \alpha\right)$, all $\lambda_{j}$ 's are either nonpositive (then replace $U$ by $-U$, and $\lambda_{j}$ by $-\lambda_{j}$ ), or nonnegative. Hence (i) is derived.

If (i) and (ii) are satisfied, one may furthermore note that, if no coordinate is essential, then $x \geqslant y$ for all $x, y$ by Lemma 2.1, and $U$ should be chosen constant, or 
all $\lambda_{j}$ 's are chosen zero. If one or more coordinates are essential, then $U$ is not constant, and $\lambda_{j}$ is zero if and only if coordinate $j$ is not essential, and $\left(\lambda_{j}\right)_{j=1}^{n}$ can be replaced by $\left(\lambda_{j}\right)_{j=1}^{n}$ if and only if there is $\alpha>0$ such that $\lambda_{j}^{\prime}=\alpha \lambda_{j}$ for all $j$. If exactly one coordinate is essential, then $U$ is ordinal, i.e., $U$ may be replaced by $U^{\prime}$ if and only if there exists increasing $\phi$ such that $U=\phi \circ U^{\prime}\left(U^{\prime}\right.$ continuous $\Leftrightarrow \phi$ continuous).

For the case where $\geqslant$ applies to a bounded subset of $\mathbb{R}^{n}$, the following result is of use.

THEOREM 2.2. Let $\geqslant$ be a binary relation on $(\sigma, \tau)^{n}$, where $-\infty \leqslant \sigma<\tau \leqslant \infty$. Then the results of Theorem 2.1 apply (with now $\geqslant$ on $(\sigma, \tau)^{n}$, and $U:(\sigma, \tau) \rightarrow \mathbb{R}$ ).

Proof. We introduce a bijective increasing (thus continuous) map $\phi: \mathbb{R} \rightarrow(\sigma, \tau)$, and define $\geqslant$ on $\mathbb{R}^{n}$ by $\left(x_{1}, \ldots, x_{n}\right) \geqslant\left(y_{1}, \ldots, y_{n}\right)$ if and only if $\left(\phi\left(x_{1}\right), \ldots, \phi\left(x_{n}\right)\right) \geqslant\left(\phi\left(y_{1}\right), \ldots, \phi\left(y_{n}\right)\right)$. Then we apply Theorem 2.1 , and define $U^{\prime}$ on $(\sigma, \tau)$ by $U^{\prime}\left(\gamma^{\prime}\right)=U(\gamma)$, where $\gamma^{\prime}=\phi(\gamma)$, for all $\gamma^{\prime}$ in $(\sigma, \tau)$. The essential point in this is that the map $\phi$ does not affect transitivity, totality, continuity, or CCI, of $\geqslant$.

\section{Decision MAKING UNDER UNCERTAINTY}

In this section we interpret the previous theorems in the context of decision making under uncertainty. Let $S=\left\{s_{1}, \ldots, s_{n}\right\}$ be a finite set, called the state space, where $n \in \mathbb{N}$. Its elements are (possible) states (of nature). Subsets of $S$ are events. Exactly one is the true state; the other states are not true. A person $T$ is uncertain which of the states is true. As an example, one may think of horse races. Of $n$ participating horses exactly one will win. By $s_{j}$ we denote the "possible state of nature" that horse $j$ will win. An element $x=\left(x_{1}, \ldots, x_{n}\right)$ of $(\sigma, \tau)^{n}$, where $-\infty \leqslant \sigma<\tau \leqslant \infty$, denotes a bet for money, that yields $\$ x_{j}$ if $s_{j}$ is the true state. Money is assumed to be a continuously divisible quantity that can take all real values between $\sigma$ and $\tau$ (also negative, if $\sigma<0$ ). By $\geqslant$ we denote the preference relation of $T$ on the set of bets. (We use the term "bet" instead of "act" in this section.) If $T$ prefers $x$ to $y$ we write $x \geqslant y$.

We assume in descriptive context, if $T$ chooses $x$ out of $\{x, y\}$, then $x \geqslant y$, and in prescriptive context, if $x \geqslant y$, then $T$ will be willing to choose $x$ out of $\{x, y\}$. We consider $\geqslant$ as the only observable entity in our model, the other entities have their meaning through $\geqslant$.

Definition. We say (SEU $=)\left[(\sigma, \tau)^{n}, \geqslant,\left(p_{j}\right)_{j=1}^{n}, U\right]$ is a Subjective Expected Utility (SEU) model (for $\geqslant$ ) if $-\infty \leqslant \sigma<\tau \leqslant \infty$, $\geqslant$ is a binary relation on $(\sigma, \tau)^{n}$, the $p_{j}$ 's are nonnegative real numbers that sum to 1 , and $U:(\sigma, \tau) \rightarrow \mathbb{R}$ is a function, such that $\left[x \geqslant y \Leftrightarrow \sum_{j=1}^{n} p_{j} U\left(x_{j}\right) \geqslant \sum_{j=1}^{n} p_{j} U\left(y_{j}\right)\right]$ for all $x, y$. Then $p_{j}$ is the subjective probability for state $s_{j}$, $U$ the (subjective) utility function (for money), and $\sum_{j=1}^{n} p_{j} U\left(x_{j}\right)$ the (subjective) expected utility for bet $x$ (under SEU). 
It is the applicability of the SEU models that we characterize in this section. We restrict our attention to the special case of continuous utility functions. One quickly observes that a necessary property for the appropriateness of an SEU model is that $\geqslant$ is CI. In the context of decision making under uncertainty CI is known under the name "Sure-thing Principle" (Savage, 1954). In Economics it is called something like "separability," such as "strong separability" in Barten and Böhm (1982), "complete strict separability" in Blackorby, Primont, and Russel (1978), or "additivity" in Katzner (1970). In Measurement Theory it is called "independence," such as in Debreu (1960), Krantz et al. (1971), and Roberts (1979), or "mutual preferential independence," Keeney and Raiffa (1976). We like most the following verbal formulation for it: the preference between bets $x$ and $y$ depends only on (their values at) the states where they differ; it is independent of (their values at) states where they are equal.

To indicate the meaning of $\mathrm{CCI}$ for decision making under uncertainty, and to show how it is related to strength of preference, consider the following.

Definition. We say $(\alpha, \beta) \geqslant_{i}^{*}(\gamma, \delta)$ if there exist $x, y$ such that $\left(x_{-i} \alpha\right) \geqslant\left(y_{-i} \beta\right)$, and $\left(x_{-i} \gamma\right) \preccurlyeq\left(y_{-i} \delta\right)$; we also say $(\alpha, \beta)>_{i}^{*}(\gamma, \delta)$ if $\geqslant$ can be replaced by $>$, or $\leqslant$ by $<$.

For the idea of this definition, suppose $\left(x_{-i} \alpha\right)>\left(y_{-i} \beta\right),\left(x_{-i} \gamma\right)<\left(y_{-i} \delta\right)$, and $\alpha>\beta, \gamma>\delta$. Then, since $\alpha>\beta$, the possibility of state $s_{i}$ seems to yield a positive argument for bet $\left(x_{-i} \alpha\right)$ as compared to bet $\left(y_{-i} \beta\right)$, assuming $T$ prefers more money to less money. If we now replace $(\alpha, \beta)$ by $(\gamma, \delta)$, then this still applies. Yet by this the preference has reversed. Since at the other states of nature nothing has changed, this reversal can only be explained by the change of matters at state $s_{i}$. Apparently the positive argument, yielded by the possibility that state $s_{i}$ is true, has lost some of its strength by the replacement. We conclude that at state $s_{i}$ the strength of preference between $(\alpha, \beta)$ exceeds that between $(\gamma, \delta)$, and write $(\alpha, \beta)>_{i}^{*}(\gamma, \delta)$. Note that this strength of preference notion has been derived from the preference relation $\geqslant$.

Of course, in general the above definition does not have to make sense, the $\geqslant *$ and $>*$ relations may have undesired properties.

DEFINITION. We say the appreciation for money (of $T$ ) has a cardinal character, independent of the state of nature, if $(\alpha, \beta) \geqslant i \quad(\gamma, \delta) \& i$ essential $\Rightarrow$ not $(\gamma, \delta)>_{j}^{*}(\alpha, \beta)$, for all $\alpha, \beta, \gamma, \delta, i, j$.

LemMA 3.1. The appreciation for money has a cardinal character, independent of the state of nature, if and only if $\geqslant$ is CCI.

Proof. Straightforward. Note:

$$
\begin{aligned}
& (\alpha, \beta) \geqslant_{i}^{*}(\gamma, \delta) \Leftrightarrow(\delta, \gamma) \geqslant_{i}^{*}(\beta, \alpha) ; \\
& (\alpha, \beta)>_{i}^{*}(\gamma, \delta) \Leftrightarrow(\delta, \gamma)>_{i}^{*}(\beta, \alpha) .
\end{aligned}
$$


THEOREM 3.1. There exists an SEU model with continuous $U$ for $\geqslant$, if and only if $\geqslant$ is a continuous weak order, and the appreciation for money of $T$ has a cardinal character, independent of the state of nature. Then $\geqslant$ is $\mathrm{CI}$; and $U$ is non-decreasing if and only if $\geqslant$ is monotone.

Proof. The first assertion follows from Lemma 3.1 and Theorem 2.2. The second is straightforward. ( $\sum_{j=1}^{n} p_{j}=1$ can always be arranged.)

In descriptive context, this characterization shows that, taking the continuity and weak order property for granted, an observed preference relation cannot be described by an SEU model if and only if $\mathrm{CCI}$ can be falsified. In normative, or prescriptive, context, it shows that, again taking continuity and weak order for granted, a person can be convinced that he should use the SEU model to determine his behavior, if and only if he can be convinced of the appropriateness of CCI.

Finally some more on strength of preference. We have only considered strength of preference w.r.t. amounts of money. One may also derive a strength of preference relation $\geqslant *$ w.r.t. bets, from $\geqslant$. This is reversed from Sarin (1982), and Theorem 7.4 of Fishburn (1970), where $\geqslant$ is derived from $\geqslant *$ by $x \geqslant y$ if $(x, y) \geqslant *(y, y)$. Deriving $\geqslant *$ from $\geqslant$ is done as follows (where we do not make explicit the assumptions that justify this, they can be found in Sarin, 1982). To see that $(x, y) \geqslant *(v, w)$ we find $\alpha, \beta, \gamma \quad$ such that $\left(y_{-1} \alpha\right) \approx x, \quad\left(w_{-1} \beta\right) \approx v, \quad$ and $\left(y_{2} \gamma\right) \approx w$. Then $(x, y) \geqslant *(v, w) \Leftrightarrow\left(\left(y_{-1} \alpha\right), y\right) \geqslant *\left(\left(w_{-1} \beta\right), w\right) \Leftrightarrow\left(\left(y_{-1,2} \alpha, \gamma\right),\left(y_{-2} \gamma\right)\right) \geqslant * \quad\left(\left(w_{-1} \beta\right)\right.$, $\left.\left(y_{-2} \gamma\right)\right) \Leftrightarrow\left(y_{-1,2} \alpha, \gamma\right) \geqslant\left(w_{-1} \beta\right)$.

\section{REFERENCES}

Barten, A. P., \& Böhm, V. (1982). Consumer theory. In K. J. Arrow \& M. D. Intriligator (Fds.), Handbook of mathematical economics (Vol. II). Amsterdam: North-Holland.

Blackorby, C., Primont, D., \& Russell, R. R. (1978). Duality, separability, and functional structure: Theory and economic applications. New York: Elsevier/North-Holland.

Blaschke, W., \& Bol, G. (1938). Geometrie der Gewebe. Berlin: Springer-Verlag.

Самасно, A. (1980). Approaches to cardinal utility. Theory and decision, 12, 359-379.

Debreu, G. (1959). Theory of value. New York: Wiley.

Debreu, G. (1960). Topological methods in cardinal utility theory. In K. J. Arrow, S. Karlin \& P. Suppes (Eds.), Mathematical methods in the social sciences, 1959: Proceedings (pp. 16-26). Stanford, CA: Stanford Univ. Press.

DE Finetri, B. (1937). La Prevision: Ses Lois Logiques, Ses Sources Subjectives. Annales de IInstitut Henri Poincaré, 7, 1-68. Translated into English in H. E. Kyburg \& H. E. Smokler (Eds.), 1964, Studies in Subjective probability (pp. 93-158) New York: Wiley.

Drèze, J. II. (1982). Decision criteria for business firms. CORE Discussion Paper 8211, Center for Operations Research \& Econometrics. Louvain-la-Neuve, 1982.

FishruRn, P. C. (1970). Utility theory for decision making. New York: Wiley.

FishbuRn, P. C. (1981). Subjective expected utility theory: A review of normative theories. Theory and Decision, 13, 139-199.

Fishburn, P. C. (1982). The foundations of expected utility. Dordrecht: Reidel.

KATZNER, D. W. (1970). Static demand theory. London: Macmillan \& Co. 
KeEnEy, R. L., \& RaIfFA, H. (1976). Decisions with multiple objectives. New York: Wiley.

Krantz, D. H., Luce, R. D., Suppes, P., \& Tversky, A. (1971). Foundations of measurement (Vol. I). New York: Academic Press.

MARSCHAK, J., \& RADNeR, R. (1972). Economic theory of teams. New Haven, CT: Yale Univ. Press.

RoBerts, F. S. (1979). Measurement theory (Encyclopedia of mathematics and its applications, Vol. 7). London: Addison-Wesley.

SARIN, R. K. (1982). Strength of preference and risky choice. Operations Research, 30, 982-997.

SAVAGE, I. J. (1954). The foundations of statistics. New York: Wiley.

SCHoemakeR, P. J. H. (1982). The expected utility model: Its variants, purposes, evidence and limitations. Journal of Economic Literature, 20, 529-563.

SEGAL, U. (1982). Expected utility theory with lotteries of bounded length (Research Memorandum No. 50). Jerusalem: Center for Research in Mathematical Economics and Game Theory, Hebrew University.

von Neumann, J., \& Morgenstern, O. (1944, 1947, 1953). Theory of games and economic behavior, Princeton, NJ: Princeton Univ. Press.

WAKKER, P. P. (1981). Agreeing probability measures for comparative probability structures. Annals of Statistics, 9, 658-663.

RECEIVED: September 23, 1982 